\title{
Poncirin suppresses lipopolysaccharide (LPS)-induced microglial inflammation and ameliorates brain ischemic injury in experimental stroke in mice
}

\author{
Li-Xuan Yang ${ }^{1}$, Fang-Yu Chen ${ }^{1}$, Hai-Long Yu ${ }^{2}$, Pin-Yi Liu ${ }^{1}$, Xin-Yu Bao ${ }^{2}$, Sheng-Nan Xia ${ }^{2}$ Yue Gu${ }^{2}$, \\ Yun $\mathrm{Xu}^{1,2,3,4,5}$, Xiang $\mathrm{Cao}^{1,2,3,4,5}$ \\ ${ }^{1}$ Department of Neurology, Nanjing Drum Tower Hospital Clinical College of Nanjing Medical University, Nanjing, China; ${ }^{2}$ Department of \\ Neurology, Drum Tower Hospital, Medical School and The State Key Laboratory of Pharmaceutical Biotechnology, Institute of Brain Science, \\ Nanjing University, Nanjing, China; ${ }^{3}$ Jiangsu Key Laboratory for Molecular Medicine, Medical School of Nanjing University, Nanjing, China; \\ ${ }^{4}$ Jiangsu Province Stroke Center for Diagnosis and Therapy, Nanjing, China; ${ }^{5}$ Nanjing Neuropsychiatry Clinic Medical Center, Nanjing, China \\ Contributions: (I) Conception and design: X Cao, Y Xu; (II) Administrative support: Y Xu; (III) Provision of study materials or patients: X Cao, Y Xu; \\ (IV) Collection and assembly of data: LX Yang, FY Chen, HL Yu, PY Liu, XY Bao, SN Xia, Y Gu, X Cao; (V) Data analysis and interpretation: LX \\ Yang, X Cao; (VI) Manuscript writing: All authors; (VII) Final approval of manuscript: All authors. \\ Correspondence to: Xiang Cao; Yun Xu. Department of Neurology, Drum Tower Hospital, Medical School and The State Key Laboratory \\ of Pharmaceutical Biotechnology, Institute of Brain Science, Nanjing University, Nanjing 210008, China. Email: xiangcao1988@163.com; \\ xuyun20042001@aliyun.com.
}

Background: Based on accumulating evidence, excessive activation of microglia-mediated inflammatory responses plays an essential role in ischemic stroke. Poncirin (Pon) exerts anti-hyperalgesic, antiosteoporotic and anti-tumor effects on various diseases. However, the roles of Pon in microglial activation and the underlying mechanism have not been elucidated. This study aimed to explore whether Pon inhibits lipopolysaccharide (LPS)-induced microglial neuroinflammation and protects against brain ischemic injury in experimental stroke in mice.

Methods: Primary microglia cells were prepared from the cerebral cortices of 1- to 2-day-old C57BL/6J mice. Murine BV2 cells and primary microglia were stimulated with LPS and the effects of a non-cytotoxic concentration of Pon on LPS-stimulated pro-inflammatory factors were measured using real-time PCR and enzyme-linked immunosorbent assays (ELISAs). Western blot analyses were used for mechanistic studies. In an in vivo study, 8-week-old male C57BL/6J mice were subjected to focal cerebral ischemia through middle cerebral artery occlusion (MCAO). Pon $(30 \mathrm{mg} / \mathrm{kg}$, i.p.) or the same volume of saline was administered after the MCAO model was established, and the infarct volume was evaluated using 2,3,5-triphenyltetrazolium chloride (TTC) staining. We also evaluated animal behaviours, the expression of pro-inflammatory cytokines and microglial activation in the ischemic hemisphere.

Results: Pon prevented the release of nitric oxide (NO), prostaglandin E2 (PGE2), interleukin (IL)-1 $\beta$, IL-6 and tumor necrosis factor-alpha (TNF- $\alpha$ ) in both BV2 cells and primary microglia stimulated with LPS. The inhibitory effects of Pon were associated with the regulation of the ERK1/2, JNK and nuclear factor kappa B (NF- $\mathrm{kB})$ signaling pathways. In mice that underwent MCAO, Pon administration decreased the lesion size and improved neurological deficits. Furthermore, Pon attenuated the production of inflammatory cytokines mainly by restraining microglial activation after ischemic stroke.

Conclusions: Based on the findings from the present study, Pon provides neuroprotection through its anti-inflammatory effects on microglia and it may be a useful treatment for ischemic stroke.

Keywords: Middle cerebral artery occlusion (MCAO); pro-inflammatory cytokines; poncirin; microglia cells; MAPK pathways 
Submitted Apr 21, 2020. Accepted for publication Sep 24, 2020.

doi: 10.21037/atm-20-3470

View this article at: http://dx.doi.org/10.21037/atm-20-3470

\section{Introduction}

Stroke, a type of cerebrovascular disease that manifests as cerebral ischemia or cerebral hemorrhage, has very high mortality and disability rates and has become the leading cause of death in China. Ischemic stroke accounts for approximately $80 \%$ of all strokes and is caused by various factors, such as hypertension, diabetes and atherosclerosis $(1,2)$. The obstruction of blood flow caused by various factors deprives the brain tissue of oxygen and glucose, which leads to irreversible neuronal apoptosis and hypoxic brain injury (3). Currently, thrombolysis and mechanical thrombectomy are the main acute treatments for ischemic stroke (2). However, less than $10 \%$ of patients with acute ischemic stroke are able to receive these therapies due to their limited treatment window and side effects. Therefore, new and effective prevention measures and safe treatments for stroke are urgently needed.

Although multiple pathological mechanisms are involved in ischemic stroke, accumulating evidence has revealed that inflammation is the main contributor to stroke progression (4,5). Microglia are the resident immune cells in the central nervous system (CNS) and function as key mediators of inflammation in the brain (6). They are derived from myeloid progenitor cells and migrate into the cerebral rudiment by embryonic day 9.5 (6). In the resting state, similar to a housekeeper, microglia continue to surveil the brain parenchyma via their long processes and protrusions (7). When the environment changes drastically, microglia promptly adopt an activated state, migrate to the injury site, engulf necrotic cells and release numerous messenger molecules of the immune response that enhance the inflammatory responses $(8,9)$. Microglial activation is necessary for host defenses, clearing debris and tissue repair, but microglial overactivation is neurotoxic. Overactivated microglia-mediated inflammatory responses contribute to neuronal injury through the secretion of various destructive mediators, such as interleukin (IL)-1 $\beta$, IL-6, nitric oxide $(\mathrm{NO})$ and tumor necrosis factor (TNF)- $\alpha$ (10). Excess NO and TNF- $\alpha$ kill oligodendrocytes, which further aggravates white matter damage under hypoxic conditions (11). Furthermore, excess cytokines exacerbate the disintegration of blood vessels (12). Thus, inhibitors that target excessive microglia activation and the inflammatory response in which it participates have become a treatment strategy for ischemic stroke.

Poncirin (Pon) is a flavanone glycoside extracted from the fruits of Poncirus trifoliata and is used as a component of traditional Chinese medicines for the treatment of asthma and inflammation (Figure 1A) (13). According to numerous studies, Pon exerts a variety of pharmacological effects on many disease models. For example, Pon relieves acetic acidinduced and formalin-induced tonic pain and prevents the infiltration of inflammatory cells (14). It promotes apoptosis in AGS cells (human gastric cancer cells) via the FasLdependent extrinsic apoptotic pathway (15). Furthermore, Pon downregulates the expression of nuclear factor of activated $\mathrm{T}$ cells $\mathrm{c} 1$ (NFATc1) to ameliorate osteoclast differentiation and bone loss (16). In addition, Pon has also been shown to possess anti-inflammatory properties. Pon and its metabolites attenuate inflammation of the lining of the colon (colitis) by preventing lipopolysaccharide (LPS) from binding to Toll-like receptors (TLRs) and inhibiting nuclear factor kappa B (NF- $\mathrm{B}$ ) activation (13). Nevertheless, the effects of Pon on the treatment of ischemic stroke related to the inhibition of microgliainduced inflammation remain unknown.

We conducted a series of in vitro and in vivo experiments to investigate the anti-inflammatory effects of Pon on microglia. BV2 cells and primary mouse microglia were used to analyze whether Pon inhibits LPS-induced inflammation and to elucidate the underlying molecular mechanism by which Pon inhibits the inflammatory response. Subsequently, we elucidated the contribution of Pon to controlling inflammation and promoting stroke recovery after ischemic injury in the brains of male C57BL/6J mice. The middle cerebral artery occlusion (MCAO) model was used as a stroke model.

We present the following article in accordance with the ARRIVE reporting checklist (available at http://dx.doi. org/10.21037/atm-20-3470).

\section{Methods}

\section{Regents and antibodies}

Pon (CAS: 14941-08-3, purity: $\geq 95 \%$ ) was purchased from ALADDIN Ltd. (Shanghai, China) and dissolved in 
A

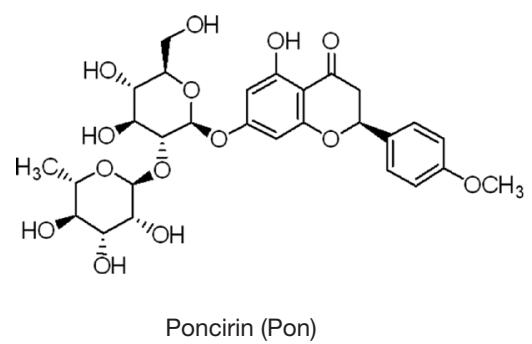

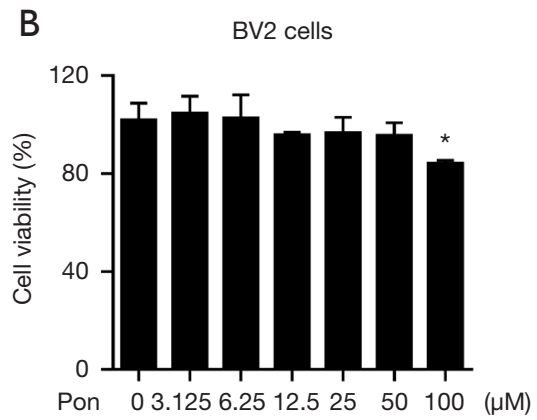

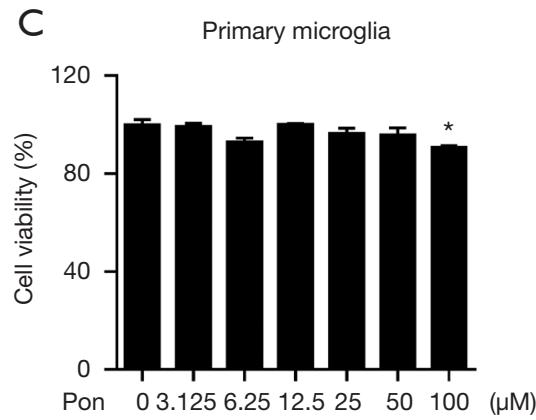

Figure 1 Effects of poncirin (Pon) on the viability of BV2 cells and primary microglia. (A) Chemical structure of Pon. (B,C) BV2 cells and primary microglia were treated with different doses of Pon $(3.125,6.25,12.5,25,50$, or $100 \mu M)$. Twenty-four hours later, the CCK8 assay was added to evaluate cell viability. The values are presented as the means \pm SEM. The data shown here are representative of three independent experiments. * $\mathrm{P}<0.05$ versus the control group.

dimethyl sulfoxide (DMSO, Amresco, Solon, OH, USA) for use in subsequent experiments. The concentration of DMSO was less than $0.1 \%$. LPS (from Escherichia coli 0111: B4) was obtained from Sigma-Aldrich (St. Louis, MO, USA). Antibodies against iNOS, ERK1/2, phosphoERK1/2, JNK, phospho-JNK, p38, phospho-p38, I $\kappa \mathrm{B} \alpha$, phospho-IкB $\alpha, \mathrm{NF}-\kappa \mathrm{B}$ p 65 and phospho-p65 were purchased from Cell Signaling Biotechnology (Hertfordshire, UK). Antibodies against GAPDH and COX2 were obtained from Bioworld Biotechnology (Minneapolis, MN, USA). The polyclonal anti-Iba1 antibody was purchased from Abcam (Cambridge, UK).

\section{Cell culture}

The murine microglia cell line BV2 was obtained from the China Infrastructure of Cell Line Resources (Beijing, China) and cultured in medium comprising 90\% DMEM (Invitrogen, Frederick, MD, USA), 10\% fetal bovine serum (FBS, Hyclone, Logan, UT, USA) and $1 \%$ antibiotics (100 U/mL penicillin and $100 \mu \mathrm{g} / \mathrm{mL}$ streptomycin) at $37^{\circ} \mathrm{C}$ in a humidified atmosphere of $5 \% \mathrm{CO}_{2}$. All experiments with BV2 cells were performed at less than passage 10 . Primary microglia were prepared from neonatal B6 mice, maintained in medium for 10-12 days, harvested by shaking for $10 \mathrm{~min}$ and reseeded in new plates. The medium used for primary microglia was the same as the medium used for BV2 cells. The purity of the primary microglia was greater than $95 \%$, as determined using Iba1 staining.

\section{Animals and a mouse model of MCAO}

Approximately 8-week-old healthy male C57BL/6J mice weighing 20-25 g were obtained from the Animal Model Center of Nanjing Medical University (Nanjing, Jiangsu, China). All animal experiments were approved by the Animal Care and Use Committee at Nanjing University (reference number: 2019AE01073) and performed according to institutional guidelines. The mice were housed in standard cages and provided with adequate food and water in a room with suitable light and temperature conditions.

All mice were randomly divided into three groups with each group consisting of 16 animals (10 mice were used to measure infarct volume and neurobehavioral deficits; 6 mice were used to detect the expression of proinflammatory factors and Iba-1) as follows: the shamoperated (sham) group; the saline-treated MCAO (MCAO + saline) group; and the $30 \mathrm{mg} / \mathrm{kg}$ Pon-treated MCAO $(\mathrm{MCAO}+\mathrm{Pon})$ group. The animals were anaesthetized with pentobarbital sodium. The MCAO mouse model was established as previously described (17). Briefly, a 6/0 monofilament nylon suture (Doccol Corporation, MA, USA) with a heat-rounded tip was inserted into the beginning of the MCA through the internal carotid artery until the ipsilateral blood flow decreased to less than 30\% of the baseline value, as monitored using laser Doppler flowmetry (Perimed Corporation, Stockholm, Sweden). After 60 min of occlusion, the filament was withdrawn to allow blood reperfusion. In the sham-operated group, the aforementioned procedure was performed, but a filament was not inserted into the MCA. After the operation, we fed the mice jelly or performed an intraperitoneal (i.p.) injection of normal saline to rehydrate. Pre-dissolved Pon or the same volume of saline was administered to each 
animal by an i.p. injection at $30 \mathrm{~min}, 24 \mathrm{~h}$ and $48 \mathrm{~h}$ after MCAO in a double-blind manner.

\section{Cell viability assays}

BV2 and primary microglia were planted in 96-well plates and treated with different concentrations of Pon for $24 \mathrm{~h}$. Then, the medium was removed, and cell viability was assessed using the Cell Counting Kit-8 (CCK-8; Dojindo Laboratories, Tokyo, Japan) according to the manufacturer's instructions. The optical density (OD) was measured at $450 \mathrm{~nm}$ with a microplate reader (Bio-Rad, Hercules, CA, USA).

\section{Nitrite analysis}

BV2 and primary microglia seeded in 24-well plates were pre-treated with Pon for $2 \mathrm{~h}$ and stimulated with LPS $(0.5 \mu \mathrm{g} / \mathrm{mL}$ for BV2 cells and $0.1 \mu \mathrm{g} / \mathrm{mL}$ for primary microglia) for $24 \mathrm{~h}$. The concentrations of $\mathrm{NO}$ in the supernatants were detected using the Griess reagent (Beyotime Biotech, Nantong, China). The OD value was measured at $540 \mathrm{~nm}$ with a microplate reader.

\section{Cytokine measurements}

BV2 and primary microglia were pre-treated with the indicated doses of Pon for $2 \mathrm{~h}$ and then stimulated with LPS $(0.5 \mu \mathrm{g} / \mathrm{mL}$ for BV2 cells and $0.1 \mu \mathrm{g} / \mathrm{mL}$ for primary microglia) for $24 \mathrm{~h}$. The supernatants were collected, and the concentrations of the cytokines PGE2, IL-1 $\beta$, IL- 6 and TNF- $\alpha$ were measured using enzyme-linked immunosorbent assays (ELISAs) according to the manufacturer's instructions (Cusabio Biotech, Wuhan, China).

\section{Real-time PCR}

Primary microglia and BV2 cells were pre-treated with Pon for $2 \mathrm{~h}$ and stimulated with LPS $(0.5 \mu \mathrm{g} / \mathrm{mL}$ for BV2 cells and $0.1 \mu \mathrm{g} / \mathrm{mL}$ for primary microglia) for $3 \mathrm{~h}$. The mice were sacrificed by cervical dislocation under anesthesia with pentobarbital sodium, and brain tissues from the penumbra were isolated. Total RNA was extracted from these cells and tissue mentioned above using TRIzol reagent (Invitrogen) according to the manufacturer's instructions. Then, the RNA was transcribed into cDNA using the PrimeScript RT Reagent Kit (Vazyme, Nanjing, China). A $20 \mu \mathrm{L}$ reaction mixture was prepared, and real-time PCR was performed using a Step One Plus PCR system (Applied Biosystems, Foster City, CA, USA) with a SYBR Green Kit (Applied Biosystems). The corresponding primers were as follows: IL-1 $\beta$, F: AAGCCTCGTGCTGTCGGACC, R: TGAGGCCCAAGGCCACAGGT; IL - 6, F : G C T G G T G A C A A C C A C G G C C T, R : AGCCTCCGACTTGTGAAGTGGT; TNF- $\alpha$, F : C A A G G A C A A G C T G C C C C G, R : GCAGGGGCTCTTGACGGCAG; GAPDH, F : G C C A A G G T G T G G G CAA G G T, R : TCTCCAGGCGGCACGTCAGA.

\section{Western blot}

Brain tissue from the ischemic penumbra and all cells were homogenized with lysis buffer (Thermo Fisher Scientific, Rockford, IL, USA) containing 1\% protease inhibitor cocktail for $30 \mathrm{~min}$. Afterwards, the mixtures were centrifuged $(15,000 \times \mathrm{g})$ at $4{ }^{\circ} \mathrm{C}$ for $15 \mathrm{~min}$. The protein concentrations in the supernatants were quantified using a BCA protein assay kit (Beyotime Biotech). Equal quantities of the proteins were denatured in SDS, electrophoresed on $8-12 \%$ SDS-PAGE gels and then transferred to PVDF membranes. The membranes were blocked with $5 \%$ skim milk for $2 \mathrm{~h}$ at room temperature, and then incubated with the appropriate primary antibodies overnight at $4{ }^{\circ} \mathrm{C}$. Subsequently, the membranes were incubated with secondary antibodies for another $2 \mathrm{~h}$, and the proteins on the membranes were detected with the ECL Detection Kit (Bioworld Biotechnology). Images were acquired using the Gel-Pro system (Tanon Technologies, Shanghai, China), and the intensity of each band was analyzed using ImageJ software (ImageJ 1.5, NIH, USA).

\section{Immunofluorescence staining}

The mice were anaesthetized and executed via cardiac perfusion with $0.9 \%$ saline followed by $4 \%$ paraformaldehyde. Their brain tissues were removed, dehydrated and sectioned into $20 \mu \mathrm{m}$ slices. The brain sections and BV2 cells were permeabilized with $0.1 \%$ Triton $\mathrm{X}-100$ for $10 \mathrm{~min}$, and blocked with 2\% BSA for 90 min. After washing 3 times with PBS, the slices and cells were incubated with primary antibodies against Iba1 (1:500) and NF-кB p65 (1:500) overnight at $4{ }^{\circ} \mathrm{C}$. The brain sections and BV2 cells were incubated with the indicated secondary antibodies for $2 \mathrm{~h}$ in the dark on the next day. DAPI $(5 \mathrm{~g} / \mathrm{mL})$ was added 
for $15 \mathrm{~min}$ to stain nuclei. Images were captured with a fluorescence microscope (Olympus BX51, Japan).

\section{TTC staining}

The mice were sacrificed by cervical dislocation at $72 \mathrm{~h}$ after the operation. Their brains were removed carefully, cut into five slices and immersed in $0.2 \%(\mathrm{w} / \mathrm{v})$ 2,3,5-triphenyltetrazolium chloride (TTC, Sigma-Aldrich) for $15 \mathrm{~min}$ for staining. The pale grey region was the infarct area, while the dark red region represented the intact brain area. Images were captured with a digital camera and analyzed using ImageJ software. The percentage of the infarct volume was calculated using the following formula: percentage of the infarct size $=($ contralateral area - ipsilateral non-infarct area $) /(2 \times$ contralateral area $)$ $\times 100 \%(18)$.

\section{Neurobehavioral tests}

The modified neurological severity score (mNSS) test was conducted on mice $72 \mathrm{~h}$ after the operation. The mNSS test comprised motor (muscle status and abnormal movement), sensory (visual, tactile, and proprioceptive), and reflex tests. Scores ranged from 0 to 18 points. Larger numbers indicated a more severe impairment in brain function.

The mice were trained on a rotarod device (RWD Life Science, Shenzhen, China) for 3 days before MCAO. All mice were trained twice a day, and each training trial lasted for $5 \mathrm{~min}$, during which the rotating rod accelerated from 4 to $40 \mathrm{rpm}$. Seventy-two hours after MCAO, the mice were placed on the device again, and the time until they fell from the rod was recorded. This test was used to quantify the motor function of the mice.

The forelimb muscle strength of the mice was assessed using the grip strength test $72 \mathrm{~h}$ after the operation (17). Each mouse was suspended by the tail, allowed to grasp the T-bar of a grip strength metre (GS3, Bioseb, France), and pulled backward in a straight line until its grip was broken. The maximum grip strength value was recorded to measure the maximum forelimb muscle strength.

\section{Statistical analysis}

SPSS 18.0 software was used to conduct statistical analysis. The normality of data distribution was analyzed by the Shapiro-Wilk test. Normally distributed data are presented as the mean \pm SEM and otherwise by the median with inter-quartile range. The normally distributed continuous variables were compared by Student's $t$-test, while nonnormally distributed variables were tested by the MannWhitney test. For multiple comparison among the groups, the data were tested using the one-way analysis variance (ANOVA) followed by Bonferroni's post-hoc test. If $\mathrm{P}<0.05$, the experimental results were considered significantly different.

\section{Results}

\section{Effects of Pon on the viability of BV2 cells and primary microglia}

Prior to evaluating the anti-inflammatory and neuroprotective activities of Pon, we examined the cytotoxicity of Pon in microglia. BV2 cells and primary microglia were treated with Pon at concentrations ranging from 0 to $100 \mu \mathrm{M}$ for $24 \mathrm{~h}$. As shown in Figure $1 B, C$, Pon did not alter cell viability at concentrations less than $50 \mu \mathrm{M}$. However, cell viability was slightly decreased when the concentration of Pon in the medium was $100 \mu \mathrm{M}$. Based on these findings, Pon was used at concentration less than $50 \mu \mathrm{M}$ in subsequent experiments.

\section{Pon attenuated the production of NO and PGE2 and the expression of $i N O S$ and COX-2 in LPS-induced microglia}

ELISAs and the Griess assay were performed to evaluate the effect of Pon on NO and PGE2 levels. LPS treatment significantly increased the levels of NO and PGE2 in BV2 cells compared with the controls. Pre-treatment with Pon $(12.5,25$, or $50 \mu \mathrm{M})$ inhibited the increase in NO and PGE2 levels in a dose-dependent manner (Figure $2 A, B)$. Similar results were obtained from primary microglia (Figure $2 C, D$ ). Considering that $\mathrm{NO}$ and PGE2 are produced by iNOS and COX-2, respectively, we subsequently evaluated the changes in the levels of the iNOS and COX-2 proteins in BV2 cells treated with Pon and LPS. Compared with LPS treatment alone, pre-treatment with Pon significantly suppressed the increased in iNOS and COX-2 protein levels in microglial cells (Figure 2E,F).

\section{Pon reduced the LPS-induced production of pro- inflammatory cytokines in BV2 cells and primary microglia}

Because microglia release various cytokines under 
A

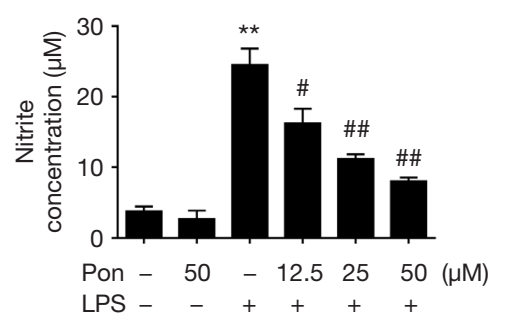

C

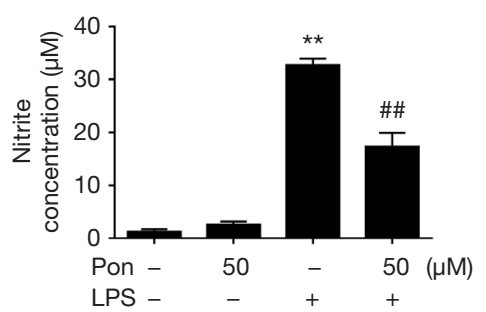

B

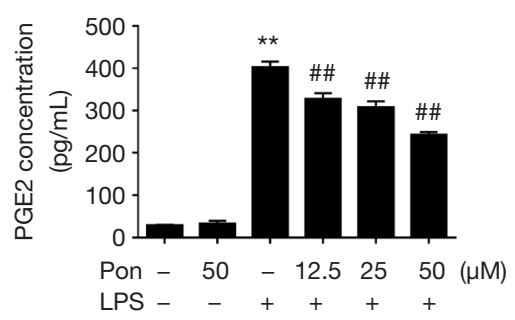

D

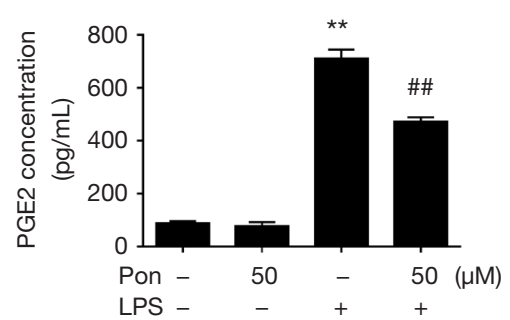

$E$

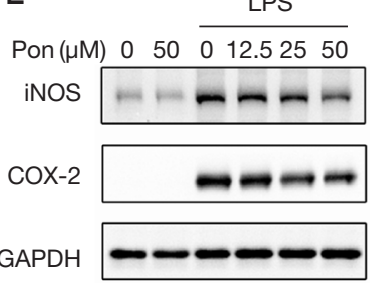

$\mathrm{F}$

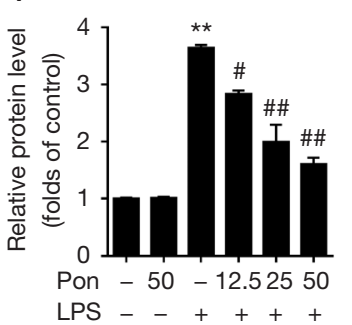

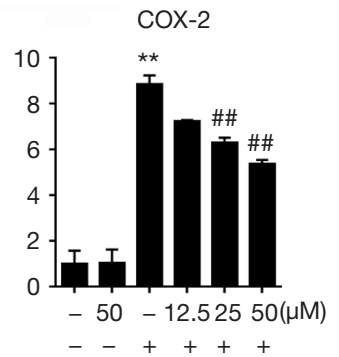

Figure 2 Effects of Pon on NO and PGE2 production and on iNOS and COX-2 expression in LPS-treated BV2 cells and primary microglia. BV2 and primary microglia were incubated with the indicated concentrations of Pon for $2 \mathrm{~h}$ and subsequently stimulated with LPS $(0.5 \mu \mathrm{g} / \mathrm{mL}$ for BV2 cells and $0.1 \mu \mathrm{g} / \mathrm{mL}$ for primary microglia) for $24 \mathrm{~h}$. (A,C) The concentrations of NO in the supernatants of BV2 cells and primary microglia were detected using the Griess reaction. (B,D) The concentrations of PGE2 in the culture medium of BV2 cells and primary microglia were detected using ELISAs. (E,F) The protein levels of iNOS and COX-2 were analyzed via Western blotting with GAPDH as an internal reference. The greyscale values of iNOS and COX-2 in the blots were quantified by Image software and normalized to GAPDH and are represented as the fold change. The values are presented as means \pm SEM. The data shown in this figure are representative of three independent experiments. ${ }^{* *}, \mathrm{P}<0.01$ versus the control group; ${ }^{\#}, \mathrm{P}<0.05$ and ${ }^{\# \#}, \mathrm{P}<0.01$ versus the LPS-treated group. Pon, poncirin; NO, nitric oxide; PGE2, prostaglandin E2; iNOS, inducible nitric oxide synthase; COX-2, cyclooxygenase-2; LPS, lipopolysaccharide; ELISA, enzyme-linked immunosorbent assay.

pathological conditions and because some of these cytokines (such as IL-1 $\beta$, IL-6 and TNF- $\alpha$ ) induce inflammatory responses that aggravate tissue injury, we evaluated whether Pon exerted an effect on the LPS-induced release of proinflammatory factors. We collected RNA and culture medium from BV2 cells and primary microglia stimulated with LPS for 3 or $24 \mathrm{~h}$, as described above. Then, we evaluated the levels of the IL-1 $\beta$, IL- 6 and TNF- $\alpha$ mRNAs and proteins using real-time PCR and ELISAs, respectively. Consistent with our hypothesis, Pon attenuated the LPS-induced increase in the expression of IL-1 $\beta$, IL-6 and TNF- $\alpha$ in BV2 cells in a dose-dependent manner (Figure $3 A, B, C, D, E, F$ ). Additionally, $50 \mu \mathrm{M}$ Pon inhibited the mRNA expression and secretion of the three proinflammatory cytokines in LPS-treated primary microglia (Figure 3G,H,I,7,K,L).

\section{Pon suppressed the phosphorylation of ERK1/2, FNK and NF- $\boldsymbol{B} B$ in LPS-activated BV2 microglial cells}

The MAPK signaling pathway is a well-known pathway involved in the inflammatory response that mainly consists of three MAPK subfamilies, including the ERK1/2, JNK and p38 families (19). Therefore, we aimed to investigate the molecular mechanism by which Pon inhibits the LPS-stimulated inflammatory response. As shown in Figure 4A, exposure of BV2 cells to LPS for $1 \mathrm{~h}$ increased the levels of the phosphorylated ERK1/2, JNK, and p38 proteins, consistent with the results of our previous study (20). When BV2 cells were pretreated with Pon, the phosphorylation of ERK1/2 and JNK, but not $\mathrm{p} 38$, was suppressed (Figure $4 A, B$ ). According to these results, we postulated that Pon inhibits inflammation by suppressing the ERK1/2 and JNK MAPK 
A

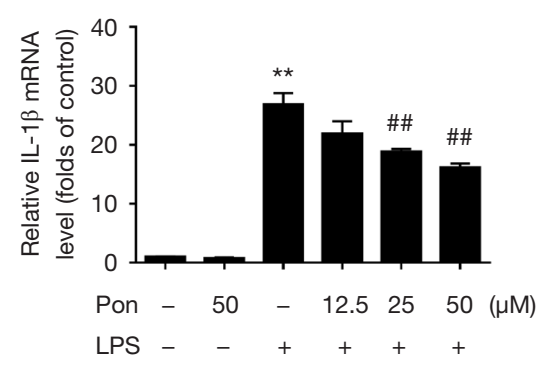

D

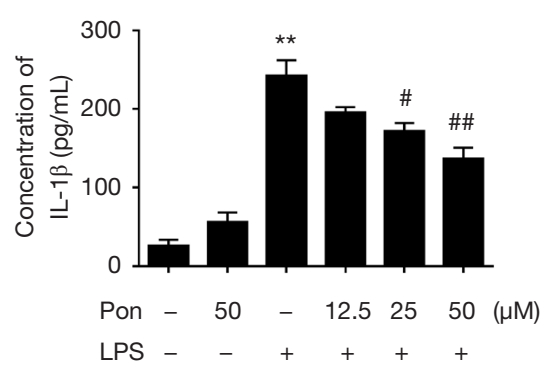

G

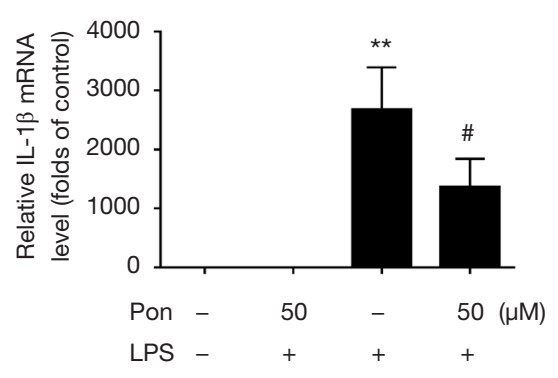

J

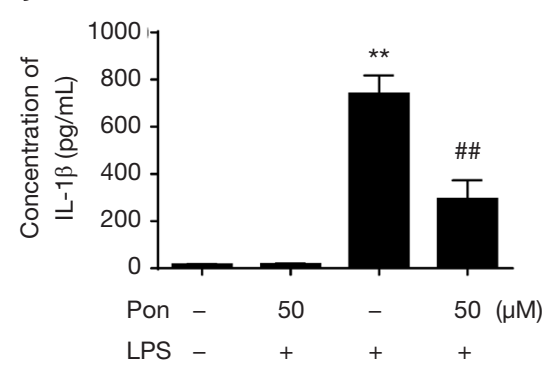

B

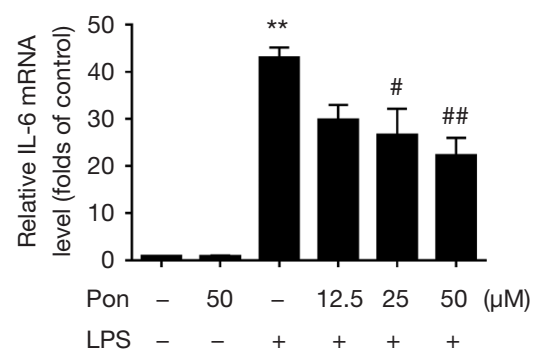

E

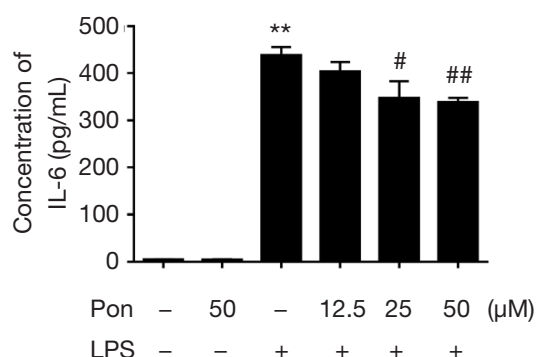

$\mathrm{H}$

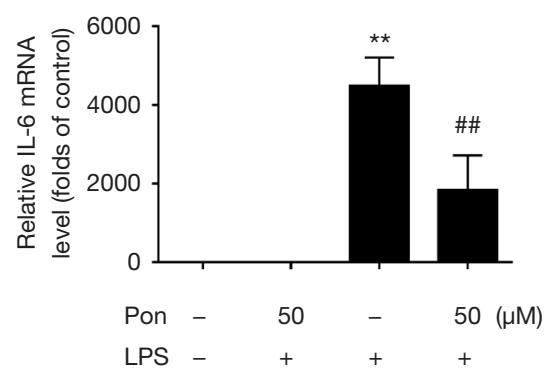

K

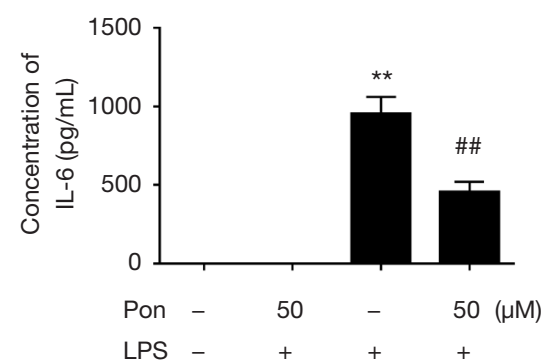

C

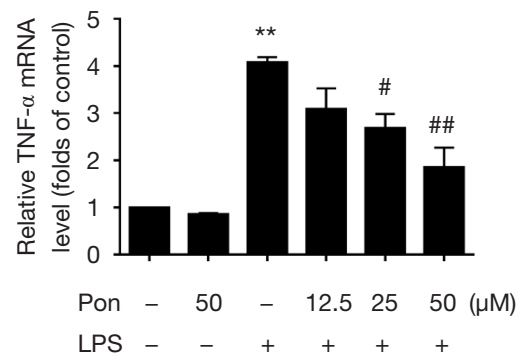

$\mathrm{F}$

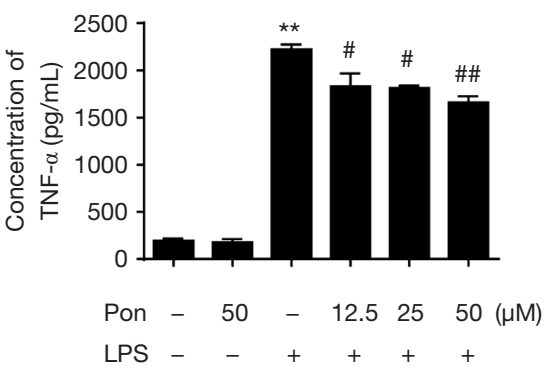

I

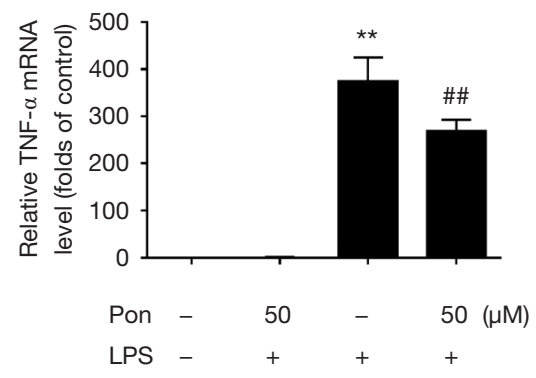

$\mathrm{L}$

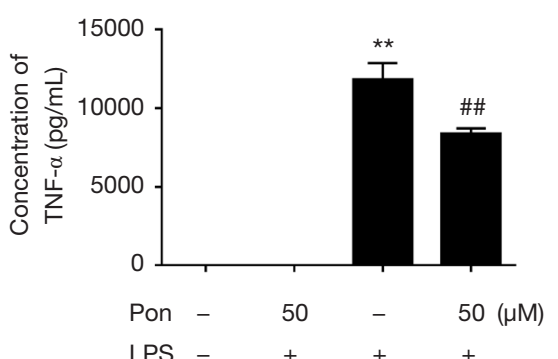

Figure 3 Effects of Pon on the expression of pro-inflammatory factors in LPS-induced BV2 cells and primary microglia. BV2 and primary microglia were treated with LPS ( $3 \mathrm{~h}$ for mRNA detection and $24 \mathrm{~h}$ for protein detection) with or without pre-treatment with the indicated concentration of Pon. (A,B,C) and (G,H,I) RNA was extracted from cells and analyzed by real-time PCR. The mRNA levels of IL-1 $\beta$, IL-6 and TNF- $\alpha$ were detected. (D,E,F) and (J,K,L) After exposure to LPS for $24 \mathrm{~h}$, the protein levels of IL-1 $\beta$, IL- 6 and TNF- $\alpha$ were evaluated by ELISA. The values are presented as mean \pm SEM. The data shown are representative of three independent experiments. ${ }^{* *}, \mathrm{P}<0.01$ versus

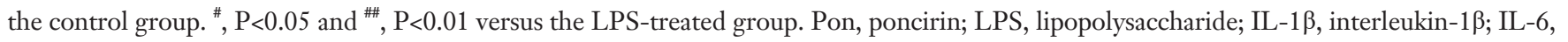
interleukin-6; TNF- $\alpha$, tumor necrosis factor-alpha; ELISA, enzyme-linked immunosorbent assay. 
A

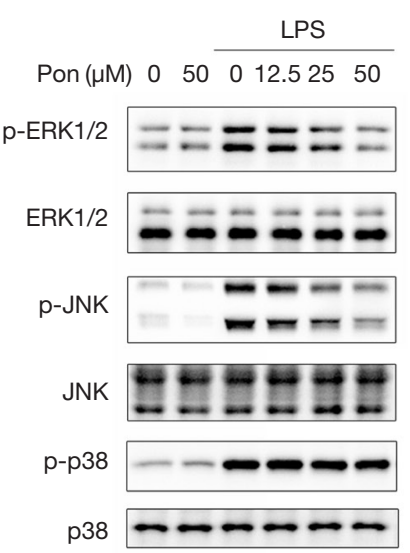

B
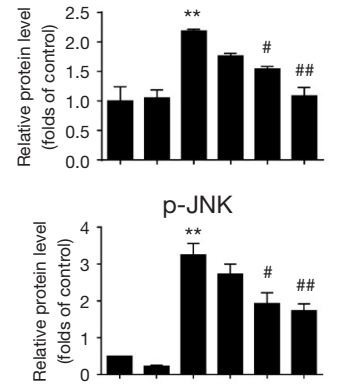

p-p38

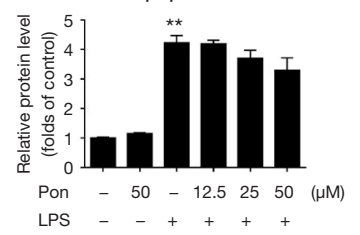

C

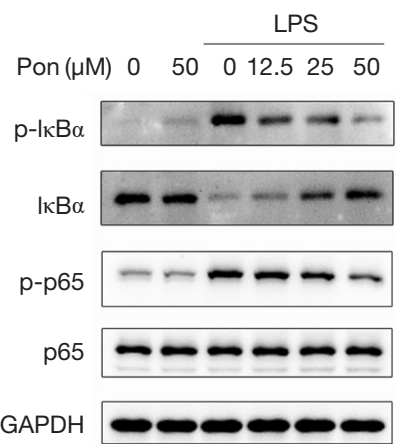

$\mathrm{D}$

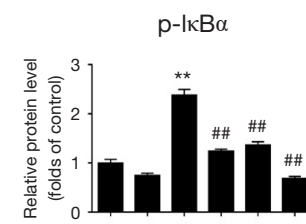

$1 \kappa \mathrm{B} \alpha$
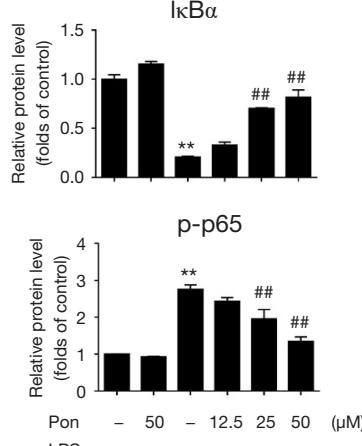

$\mathrm{E}$
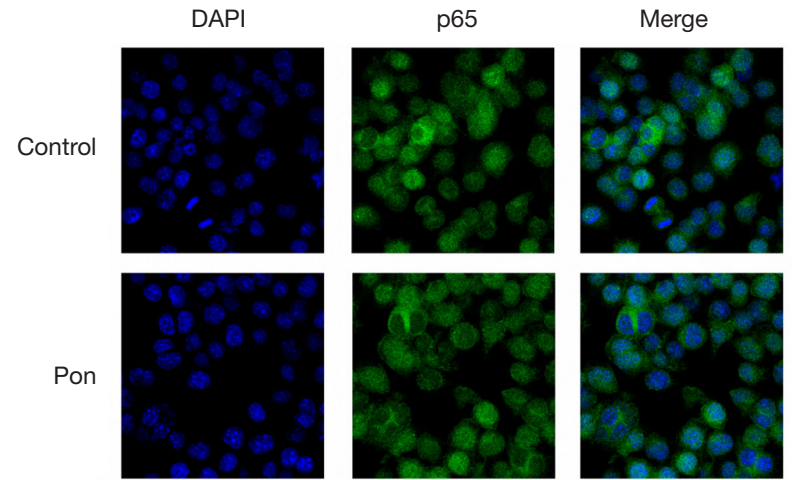

DAPI
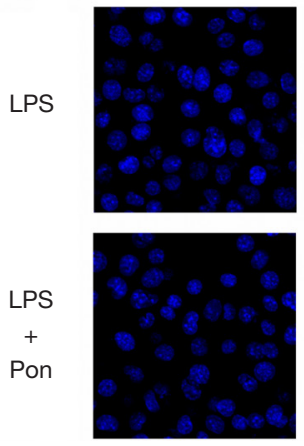

p65
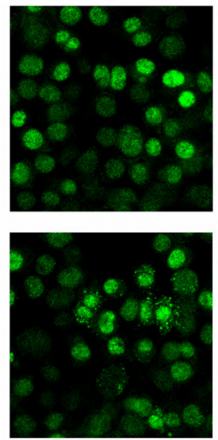

Merge
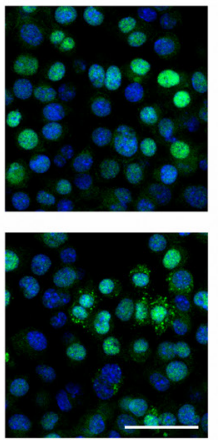

Figure 4 Effects of Pon on the LPS-induced activation of the MAPK and NF/ $/ \mathrm{B}$ pathways in microglial cells. (A,B) BV2 cells were incubated with or without Pon $(12.5,25$, or $50 \mu \mathrm{M})$ for $2 \mathrm{~h}$ prior to LPS $(0.5 \mu \mathrm{g} / \mathrm{mL})$ exposure. Then, the cell lysates were collected and subjected to Western blot analysis to detect the protein levels of ERK1/2, JNK, p38, p-ERK1/2, p-JNK and p-p38 in BV2 cells. (C,D) The expression of $\mathrm{p}-\mathrm{I} \kappa \mathrm{B} \alpha, \mathrm{I} \mathrm{\kappa} \mathrm{B} \alpha, \mathrm{p} 65$ and $\mathrm{p}-\mathrm{p} 65$ in BV2 cells was analyzed by Western blotting. The greyscale value of each band was evaluated by ImageJ software. (E) BV2 cells were fixed with 4\% paraformaldehyde for $30 \mathrm{~min}$, permeabilized with $0.1 \%$ Triton X-100 for $10 \mathrm{~min}$, blocked with $2 \%$ BSA for 90 min, and incubated overnight with primary antibodies against NF- $\mathrm{kB}$ p 65 . After incubation with the secondary antibody and DAPI, immunofluorescence microscopy was used to observe the localization of NF-kB p65 (green) and the nuclei (blue, 400x). Scale bar represents $50 \mu \mathrm{m}$. The values are presented as mean \pm SEM. The data shown are representative of three independent experiments. **, $\mathrm{P}<0.01$ versus the control group. ${ }^{\#}, \mathrm{P}<0.05$ and ${ }^{\# \#}, \mathrm{P}<0.01$ versus the LPS-treated group. Pon, poncirin; LPS, lipopolysaccharide; MAPK, mitogen-activated protein kinase; NF- $\mathrm{\kappa B}$, nuclear factor kappa B.

signaling pathways without altering the activation of $\mathrm{p} 38$ MAPK.

The NF- $\mathrm{KB}$ family is a family of transcription factors that responds quickly to inflammation and tissue injury (21). Proteins of the inhibitory $\kappa \mathrm{B}$ (I $\kappa \mathrm{B})$ family inhibit NF- $\kappa \mathrm{B}$, particularly dimers containing the p65 or c-Rel subunit (22).

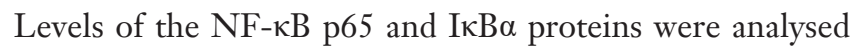
in BV2 cells treated with LPS for $1 \mathrm{~h}$ using Western blotting to investigate the effect of Pon on the NF- $\mathrm{KB}$ signalling pathway. LPS treatment significantly increased the level of phosphorylated NF-кB p65, while Pon suppressed this phosphorylation in a dose-dependent 
manner. I $\mathrm{B} \alpha$ was markedly phosphorylated and degraded in BV2 cells after LPS stimulation. However, pre-treatment with Pon significantly decreased the degradation and phosphorylation of I $\kappa \mathrm{B} \alpha$ (Figure $4 C, D$ ). The inhibitory effect of Pon on the activation of p65 was confirmed by the results of immunofluorescence staining. NF- $\mathrm{kB}$ p65 was mainly located in the cytoplasm in the absence of LPS and in the presence of Pon alone, whereas the LPS treatment markedly increased the fluorescence intensity of NF- $\kappa \mathrm{B}$ p 65 in the nucleus. Pre-treatment with Pon significantly blocked the translocation of NF- $\mathrm{kB}$ p 65 from the cytoplasm to the nucleus (Figure 4E). Thus, we propose that Pon inhibits inflammatory responses through the $\mathrm{NF}-\kappa \mathrm{B}$ signaling pathway.

\section{Pon decreased the infarct volume and improved neurological deficits after ischemic stroke}

To investigate whether the in vitro anti-inflammatory effects of Pon are associated with therapeutic activities in vivo, an experimental mouse model of ischemic stroke in which neuroinflammation is the main pathogenic event was used. We did not find any studies that mentioned the use of Pon in stroke research. However, Pon $(30 \mathrm{mg} / \mathrm{kg})$ markedly reduces pain behaviors in experimental models of inflammatory pain (14). Thus, we chose $30 \mathrm{mg} / \mathrm{kg}$ Pon for the in vivo study. We excluded two mice that died within $72 \mathrm{~h}$ after MCAO and replaced them with another two mice. TTC staining was performed to analyze infarct volume, and no infarction was detected in the sham group. In addition, the infarct volume was significantly decreased in mice that received Pon via an i.p. injection at $30 \mathrm{~min}, 24 \mathrm{~h}$ and $48 \mathrm{~h}$ after MCAO compared with the vehicle-treated MCAO group $(17.41 \% \pm 2.50 \%$ in the group treated with MCAOPon vs. $24.95 \% \pm 1.85 \%$ in the group treated with MCAOsaline at $72 \mathrm{~h}, \mathrm{P}<0.05, \mathrm{n}=10$, Figure $5 A, B)$. Subsequently, we evaluated changes in brain function deficits in each group. Consistent with a smaller infarct size, Pon led to a lower mNSS score, increased motor function and stronger grip strength (mNSS: $6.60 \pm 0.50$ points in the group treated with MCAO-Pon vs. 9.60 \pm 0.54 points in the group treated with MCAO-saline at $72 \mathrm{~h}, \mathrm{P}<0.01$; rotarod test: $154.50 \pm 21.36$ for the group treated with MCAO-Pon vs. $80.86 \pm 14.65$ for the group treated with $\mathrm{MCAO}$-saline at $72 \mathrm{~h}, \mathrm{P}<0.05$; grip strength test: $72.11 \pm 7.83$ for the group treated with MCAO-Pon vs. $45.75 \pm 7.09$ for the group treated with MCAO-saline at $72 \mathrm{~h}, \mathrm{P}<0.05, \mathrm{n}=10$, Figure $5 C, D, E)$. Based on these results, Pon attenuates ischemic brain injury and behavioral deficits in a mouse model of experimental stroke.

\section{Pon attenuated the production of inflammatory cytokines by restraining microglial activation after ischemic stroke}

Microglia are usually activated after tissue injury and activated microglia aggravate the inflammatory response by simultaneously releasing many pro-inflammatory factors (12). To investigate whether the neuroprotective effects of Pon are related to the inhibition of microglial activation and pro-inflammatory factor production, we further assessed the levels of the IL- $1 \beta$, IL- 6 and TNF- $\alpha$ mRNA in tissues from the ischemic hemisphere at 3 days after MCAO. The mRNA levels of these three proinflammatory cytokines were increased in the MCAO-saline group compared with the sham group, but this elevation was reversed by the Pon injection (Figure $6 A, B, C$ ). Finally, we detected the expression of Iba1, an indicator of microglial activation, in the ischemic hemisphere 3 days after MCAO by performing a Western blot analysis. Compared with saline treatment, Pon significantly suppressed the expression of Iba 1 after MCAO (Figure 6D,E). Afterwards, we evaluated the degree of microglial activation in the ischemic penumbra 3 days after MCAO using immunofluorescence staining (Figure 6F). The microglia activation caused by cerebral ischemic injury was attenuated by Pon as well. Thus, Pon decreases the infarct volume and improves neurological deficits after ischemic stroke, which might be the result of the modulation of microglial activation and subsequent inflammatory responses.

\section{Discussion}

Ischemic stroke is a global public health issue, and its high morbidity and mortality rates are closely related to the severity of ischemic brain injury (1). The pathological mechanisms of ischemic brain injury are very complicated and involve many factors. Among them, the microgliamediated inflammatory response participates in the entire process of this disease and is one of the most important factors affecting the prognosis of patients with ischemic stroke (4). In the present study, pre-treatment with Pon effectively inhibited the LPS-induced production of proinflammatory mediators in BV2 microglial cells and primary microglia by regulating the activation of the MAPK and $\mathrm{NF}-\mathrm{KB}$ signaling pathways. According to our in vivo data, the i.p. injection of Pon attenuated the brain infarct volume and improved neurological deficits induced by transient 
A

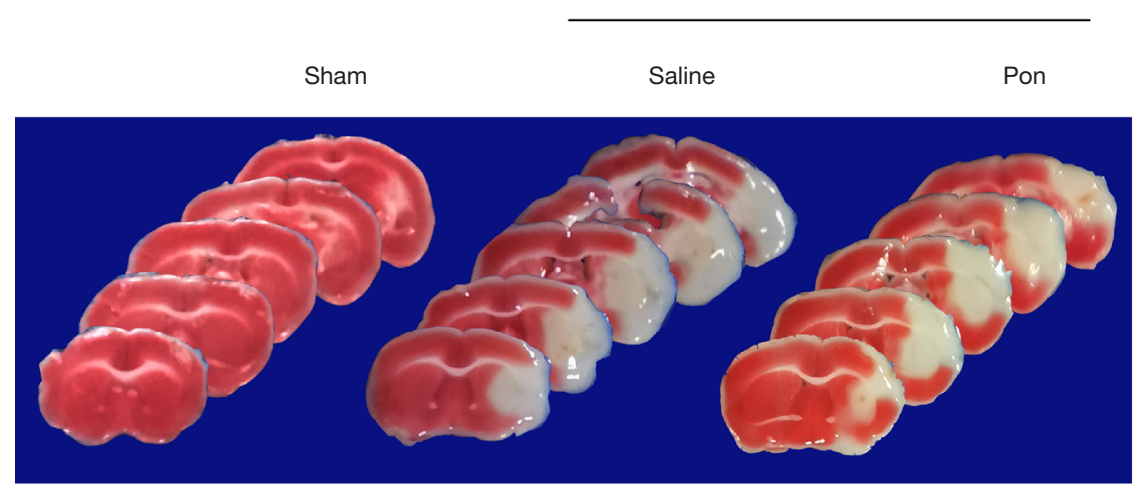

C

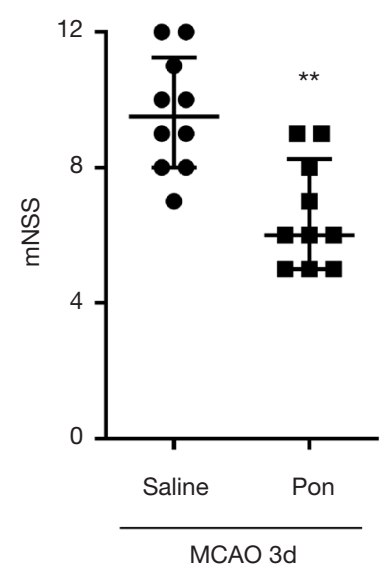

MCAO 3d

D

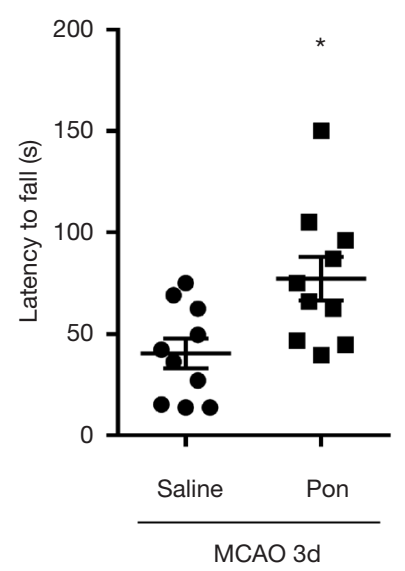

B

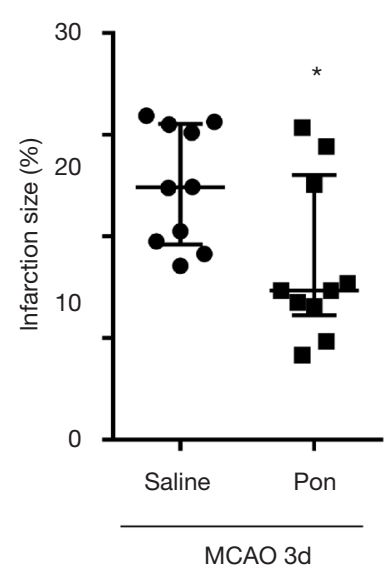

E

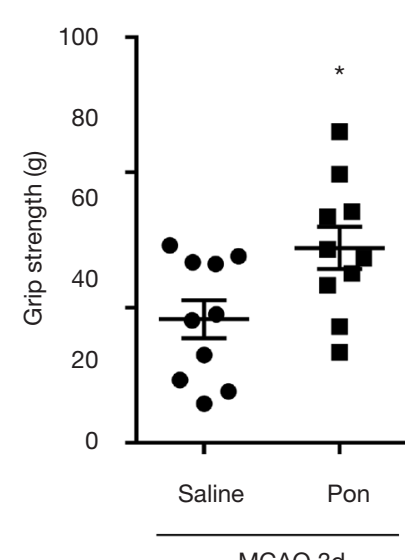

Figure 5 Effects of Pon on the infarct size and neurological function in mice after focal cerebral ischemia. Mice were subjected to MCAO and administered Pon (30 mg/kg, once a day for 3 days) or saline via an i.p. injection. (A) Representative images of brain sections stained with TTC 3 days after MCAO; (B) infarct volume ( $\mathrm{n}=10)$; (C) the mNSS scores; (D) the results of the rotarod test; (E) the results of the grip strength test $(n=10)$. (B,C) The values are performed by the median with inter-quartile range. (D,E) The values are presented as mean \pm SEM. *, $\mathrm{P}<0.05$ and **, $\mathrm{P}<0.01$ versus the MCAO-saline group. Pon, poncirin; MCAO, middle cerebral artery occlusion; TTC, 2,3,5-triphenyltetrazolium chloride; $\mathrm{mNSS}$, modified neurological severity score.

focal ischemia. Furthermore, Pon significantly suppressed microglial activation in the cerebral cortical penumbra and the release of inflammatory cytokines, suggesting that the protective effects of Pon on experimentally induced stroke were at least partially mediated by the attenuation of microglia-mediated neuroinflammation. Taken together, Pon potentially represents a neuroprotective agent to treat ischemic brain injury.
NO and PGE2 produced by iNOS and COX-2 are two of the main proteins involved in post-ischemic inflammation (23). Importantly, iNOS is expressed in the human brain after ischemic infarction (24). Inhibition of iNOS activity by its inhibitor aminoguanidine results in large decreases in infarct volume in MCAO models (25). Likewise, COX-2 inhibition by specific inhibitors and COX-2 knockout in mice result in a significant reduction 
A
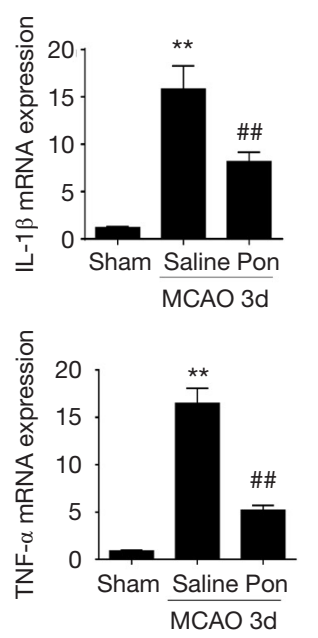

$\mathrm{D}$

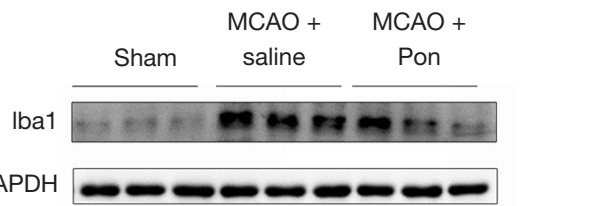

B

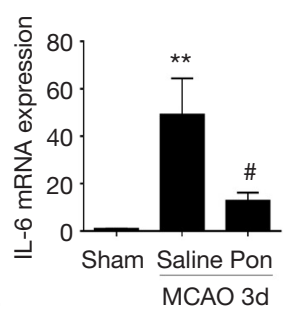

$\mathrm{E}$

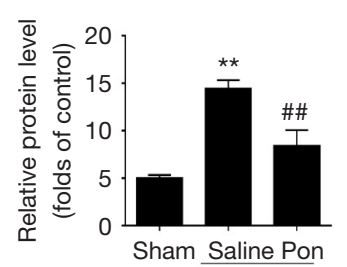

MCAO 3d

$\mathrm{F}$
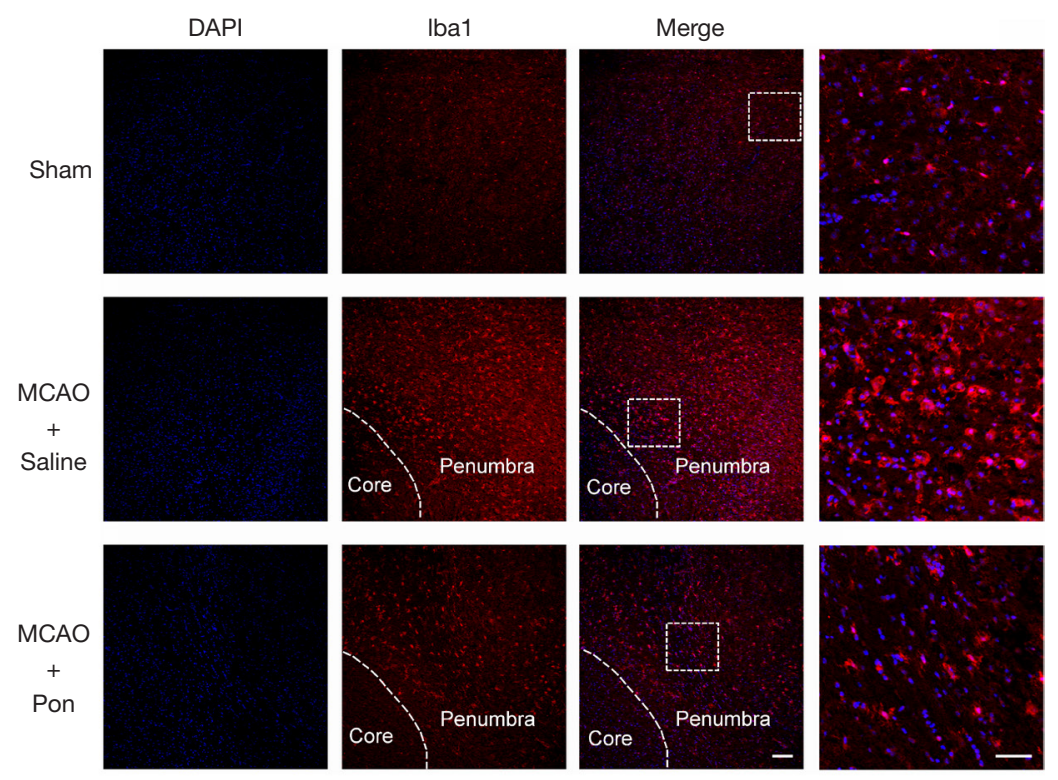

Figure 6 Effects of Pon on the production of inflammatory cytokines and microglial activation in mice after focal cerebral ischemia. Mice were subjected to MCAO and administered Pon or saline via i.p. injection. (A,B,C). The mRNA levels of IL-1 $\beta$, IL-6 and TNF- $\alpha$ in semiinfarcted tissue were assessed by real-time PCR $(n=6)$. $(D, E)$ Western blot analysis of Iba1 expression in the ischemic penumbras of sham and MCAO model mice after $72 \mathrm{~h}(\mathrm{n}=6)$. (F) Representative images of tissue sections from the ischemic penumbra collected $72 \mathrm{~h}$ after MCAO and stained with Iba1 (red) and DAPI (blue, scale bar $=100$ and $50 \mu \mathrm{m}$ ). The values are presented as mean $\pm \mathrm{SEM}$. **, $\mathrm{P}<0.01$ versus the sham group. ", $\mathrm{P}<0.05$ and ${ }^{\#}, \mathrm{P}<0.01$ versus the MCAO-saline groups. Pon, poncirin; MCAO, middle cerebral artery occlusion; IL-1 $\beta$, interleukin-1 $\beta$; IL-6, interleukin-6; TNF- $\alpha$, tumor necrosis factor-alpha.

in brain injury produced by focal ischemia $(26,27)$. In the present study, pre-treatment with Pon dose-dependently decreased NO and PGE2 production and inhibited iNOS and COX-2 expression at the protein level in LPS-induced microglia (Figure 2). These observations are consistent with those of previous studies describing the anti-inflammatory effects of Pon on ethanol-induced gastric damage in mice (28).

IL-1 $\beta$, IL- 6 and TNF- $\alpha$ are likely the most extensively studied pro-inflammatory cytokines involved in stroke. The levels of IL-1 $\beta$, IL- 6 and TNF- $\alpha$ are significantly increased by many folds (up to 40 -fold) in the brain within the first $24 \mathrm{~h}$ after the induction of focal cerebral ischemia in mice (29). These three cytokines are mainly synthesized by microglia in the stroke-lesioned rodent brain (30). In a mouse model of experimental stroke, the administration of neutralizing antibodies against IL- $1 \beta$ and TNF- $\alpha$ reduce the infarct volume after MCAO, while a striatal injection of IL- $1 \beta$ exacerbates ischemic damage (30,31). IL-6 is a pleiotropic pro-inflammatory cytokine, and the role of IL-6 in ischemic stroke is controversial. Several studies have reported strong neuroprotective effects of IL-6 inhibition, while Loddick et al. found that intracerebroventricular injection of recombinant IL-6 significantly reduced ischemic brain damage after MCAO (32-34). In the present study, we confirmed that Pon not only suppresses the LPS-induced expression of the IL-1 $\beta$, IL- 6 and TNF- $\alpha$ mRNA and proteins in microglia, but also inhibits the production of these pro-inflammatory cytokines in the mouse brain after MCAO (Figures 3,6).

Several studies have reported that the MAPK signaling pathways, including the ERK1/2, JNK and p38 pathways, play vital roles in microglial activation and inflammatory reactions (19). Inhibition of phospho-ERK1/2 and p38 in activated microglia by two specific kinase inhibitors (PD98059 and SB203580) almost completely reduces the 
production of NO and TNF- $\alpha$ (35). JNKs are essential mediators of relevant pro-inflammatory functions in microglia (36). Luteolin, a flavonoid, has been shown to inhibit LPS-induced IL-6 production in the brain by inhibiting the JNK pathway in microglia (32). Thus, treatments that inhibit MAPKs may be a promising intervention for inflammatory responses induced by overactivated microglia. In our current study, we confirmed that these three MAPK signaling pathways were involved in microglial activation and that LPS-stimulated phosphorylation of ERK $1 / 2$ and JNK was markedly diminished in BV2 microglia after pre-treatment with Pon, suggesting that ERK1/2 and JNK are two important molecular targets of Pon (Figure 4). Furthermore, Pon tended to decrease the levels of phospho-p38. However, a study performed by Chun et al. suggested that Pon inhibits receptor activator of NF- $\mathrm{NB}$ ligand (RANKL)-induced JNK activation without substantially altering the levels of phosphorylated p38 and ERK in RAW 264.7 cells, which are murine pre-osteoclasts/macrophages. The discrepancy in phospho-MAPK expression may result from the use of different cell types and experimental conditions.

In addition to MAPK pathways, NF- $\kappa \mathrm{B}$ activation is also critically required for microglia-mediated CNS inflammation (21). In the resting state, NF- $\kappa B$ is sequestered in the cytoplasm by binding with the inhibitory protein ІкB. Upon activation by many stimuli, IкBs are phosphorylated and degraded by ubiquitination. I $\kappa$ B degradation leads to the phosphorylation of the NF- $\mathrm{\kappa B}$ p65 subunit and its translocation into the nucleus to induce the transcription of several pro-inflammatory mediators. As shown in a recent study by Ganbold et al., NF- $\kappa \mathrm{B}$ p65 silencing decreases the expression of pro-inflammatory cytokines and facilitates the anti-inflammatory polarization of microglia (37). Overexpression of heat shock protein 70 (HSP70) in microglia has been reported to protect against focal and global cerebral ischemia by preventing I $\mathrm{B}$ phosphorylation (38). Pon has been shown to inhibit NF- $\kappa$ B in RAW 264.7 macrophages exposed to LPS (39). Consistent with these results, we unequivocally demonstrated that pre-treatment with Pon significantly reduced LPS-induced I $\mathrm{L} B \alpha$ phosphorylation and degradation in microglia. Subsequently, Pon suppressed NF- $\mathrm{KB}$ p 65 activation and nuclear translocation, suggesting

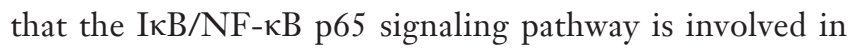
the inhibitory effects of Pon on the overexpression of proinflammatory mediators in LPS-treated microglia (Figure 4).

The MCAO model is the most commonly used animal model in studies of ischemic brain injury, and one of the most prominent features of ischemic stroke is neuroinflammation mediated by activated microglia. In follow-up experiments, we observed the neuroprotective effect of Pon on the MCAO model. Our results showed that Pon administration at $30 \mathrm{~min}, 24 \mathrm{~h}$ and $48 \mathrm{~h}$ after MCAO obviously attenuated the brain infarct size and neurological deficits on day 3 after MCAO (Figure 5). Moreover, Pon reduced the expression of Iba1, an indicator of microglial activation, suggesting that Pon exerts its potential neuroprotective effect by suppressing inflammation caused by microglia activation (Figure 6). Nevertheless, to reduce the use of experimental animals, the number of mice in each group was not large which may be a potential source of bias. Increasing evidence in recent years has shown that activated microglia can be divided into pro-inflammatory (M1) and anti-inflammatory (M2) phenotypes. As shown in our previous study, M1 and M2 microglia can switch phenotypes during the process of stroke (40). Malibatol A, a natural resveratrol oligomer, protects against focal cerebral ischemia-reperfusion injury by modulating microglial polarization from the M1 to the M2 phenotype (41). Thus, treatments that inhibit overactivated pro-inflammatory M1 microglia by switching them to the protective M2 phenotype has been suggested as a potential therapeutic strategy for ischemic stroke. Further experiments will study the effect of Pon on microglial polarization in in vivo and in vitro mouse models of ischemic stroke to make our research more meaningful and interesting.

\section{Conclusions}

In summary, Pon exerts anti-inflammatory and neuroprotective effects on LPS-induced inflammatory responses in microglia and ischemic injury in the brain of a mouse model experimentally induced stroke. These findings suggest that Pon may represent a potential treatment for ischemic stroke.

\section{Acknowledgments}

Funding: This study was supported by the National Natural Science Foundation of China (No. 81701170, 81630028,81920108017 and 81571134), the Natural Science Foundation of Jiangsu Province of China (No. BK20170122), the Young Talent Support Program from Jiangsu Association for Science and Technology and Jiangsu Province Key Medical Discipline (No. ZDXKA2016020). 


\section{Footnote}

Reporting Checklist: The authors have completed the ARRIVE reporting checklist. Available at http://dx.doi. org/10.21037/atm-20-3470

Data Sharing Statement: Available at http://dx.doi. org/10.21037/atm-20-3470

Conflicts of Interest: All authors have completed the ICMJE uniform disclosure form (available at http://dx.doi. org/10.21037/atm-20-3470). The authors have no conflicts of interest to declare.

Ethical Statement: The authors are accountable for all aspects of the work in ensuring that questions related to the accuracy or integrity of any part of the work are appropriately investigated and resolved. All animal experiments were approved by the Animal Care and Use Committee at Nanjing University (reference number: 2019AE01073) and performed according to institutional guidelines.

Open Access Statement: This is an Open Access article distributed in accordance with the Creative Commons Attribution-NonCommercial-NoDerivs 4.0 International License (CC BY-NC-ND 4.0), which permits the noncommercial replication and distribution of the article with the strict proviso that no changes or edits are made and the original work is properly cited (including links to both the formal publication through the relevant DOI and the license). See: https://creativecommons.org/licenses/by-nc-nd/4.0/.

\section{References}

1. Phipps MS, Cronin CA. Management of acute ischemic stroke. BMJ 2020;368:16983.

2. Stoll G, Nieswandt B. Thrombo-inflammation in acute ischaemic stroke - implications for treatment. Nat Rev Neurol 2019;15:473-81.

3. Radak D, Katsiki N, Resanovic I, et al. Apoptosis and Acute Brain Ischemia in Ischemic Stroke. Curr Vasc Pharmacol 2017;15:115-22.

4. Salter MW, Stevens B. Microglia emerge as central players in brain disease. Nat Med 2017;23:1018-27.

5. Hu X, Leak RK, Shi Y, et al. Microglial and macrophage polarization-new prospects for brain repair. Nat Rev Neurol 2015;11:56-64.
6. Tay TL, Savage JC, Hui CW, et al. Microglia across the lifespan: from origin to function in brain development, plasticity and cognition. J Physiol 2017;595:1929-45.

7. Nimmerjahn A, Kirchhoff F, Helmchen F. Resting microglial cells are highly dynamic surveillants of brain parenchyma in vivo. Science 2005;308:1314-8.

8. Neher JJ, Emmrich JV, Fricker M, et al. Phagocytosis executes delayed neuronal death after focal brain ischemia. Proc Natl Acad Sci U S A 2013;110:E4098-107.

9. Michelucci A, Heurtaux T, Grandbarbe L, et al. Characterization of the microglial phenotype under specific pro-inflammatory and anti-inflammatory conditions: Effects of oligomeric and fibrillar amyloidbeta. J Neuroimmunol 2009;210:3-12.

10. Xu L, He D, Bai Y. Microglia-Mediated Inflammation and Neurodegenerative Disease. Mol Neurobiol 2016;53:6709-15.

11. Merrill JE, Ignarro LJ, Sherman MP, et al. Microglial cell cytotoxicity of oligodendrocytes is mediated through nitric oxide. J Immunol 1993;151:2132-41.

12. Qin C, Zhou LQ, Ma XT, et al. Dual Functions of Microglia in Ischemic Stroke. Neurosci Bull 2019;35:921-33.

13. Kang GD, Kim DH. Poncirin and its metabolite ponciretin attenuate colitis in mice by inhibiting LPS binding on TLR4 of macrophages and correcting Th17/ Treg imbalance. J Ethnopharmacol 2016;189:175-85.

14. Afridi R, Khan AU, Khalid S, et al. Anti-hyperalgesic properties of a flavanone derivative Poncirin in acute and chronic inflammatory pain models in mice. BMC Pharmacol Toxicol 2019;20:57.

15. Saralamma VV, Nagappan A, Hong GE, et al. Poncirin Induces Apoptosis in AGS Human Gastric Cancer Cells through Extrinsic Apoptotic Pathway by up-Regulation of Fas Ligand. Int J Mol Sci 2015;16:22676-91.

16. Chun KH, Jin HC, Kang KS, et al. Poncirin Inhibits Osteoclast Differentiation and Bone Loss Through DownRegulation of NFATc1 In Vitro and In Vivo. Biomol Ther (Seoul) 2020;28:337-43.

17. Liu PY, Zhang Z, Liu Y, et al. TMEM16A Inhibition Preserves Blood-Brain Barrier Integrity After Ischemic Stroke. Front Cell Neurosci 2019;13:360.

18. Zhang Z, Guo MD, Liu Y, et al. RNPS1 inhibition aggravates ischemic brain injury and promotes neuronal death. Biochem Biophys Res Commun 2020;523:39-45.

19. Ji RR, Gereau RW, Malcangio M, et al. MAP kinase and pain. Brain Res Rev 2009;60:135-48.

20. Weng L, Zhang H, Li XX, et al. Ampelopsin attenuates 


\section{Page 14 of 14}

lipopolysaccharide-induced inflammatory response through the inhibition of the NF- $\kappa$ B and JAK2/STAT3 signaling pathways in microglia. Int Immunopharmacol 2017;44:1-8.

21. Sochocka M, Diniz BS, Leszek J. Inflammatory Response in the CNS: Friend or Foe? Mol Neurobiol 2017;54:8071-89.

22. Wang Q, Zhou X, Yang L, et al. Gentiopicroside (GENT) protects against sepsis induced by lipopolysaccharide (LPS) through the NF-kappaB signaling pathway. Ann Transl Med 2019;7:731.

23. Caso JR, Pradillo JM, Hurtado O, et al. Toll-like receptor 4 is involved in subacute stress-induced neuroinflammation and in the worsening of experimental stroke. Stroke 2008;39:1314-20.

24. Forster C, Clark HB, Ross ME, et al. Inducible nitric oxide synthase expression in human cerebral infarcts. Acta Neuropathol 1999;97:215-20.

25. Iadecola C, Zhang F, Xu X. Inhibition of inducible nitric oxide synthase ameliorates cerebral ischemic damage. Am J Physiol 1995;268:R286-92.

26. Iadecola C, Niwa K, Nogawa S, et al. Reduced susceptibility to ischemic brain injury and $\mathrm{N}$-methyl-Daspartate-mediated neurotoxicity in cyclooxygenase-2deficient mice. Proc Natl Acad Sci U S A 2001;98:1294-9.

27. Carlson NG. Neuroprotection of cultured cortical neurons mediated by the cyclooxygenase- 2 inhibitor APHS can be reversed by a prostanoid. J Neurosci Res 2003;71:79-88.

28. Kang GD, Kim DH. Ponciretin attenuates ethanolinduced gastric damage in mice by inhibiting inflammatory responses. Int Immunopharmacol 2017;43:179-86.

29. Hill JK, Gunion-Rinker L, Kulhanek D, et al. Temporal modulation of cytokine expression following focal cerebral ischemia in mice. Brain Res 1999;820:45-54.

30. Lambertsen KL, Biber K, Finsen B. Inflammatory cytokines in experimental and human stroke. J Cereb Blood Flow Metab 2012;32:1677-98.

31. Stroemer RP, Rothwell NJ. Exacerbation of ischemic brain damage by localized striatal injection of interleukin-1beta in the rat. J Cereb Blood Flow Metab 1998;18:833-9.

Cite this article as: Yang LX, Chen FY, Yu HL, Liu PY, Bao XY, Xia SN, Gu Y, Xu Y, Cao X. Poncirin suppresses lipopolysaccharide (LPS)-induced microglial inflammation and ameliorates brain ischemic injury in experimental stroke in mice. Ann Transl Med 2020;8(21):1344. doi: 10.21037/atm-203470

\section{Yang et al. Poncirin suppresses inflammation in ischemic stroke}

32. Jang S, Kelley KW, Johnson RW. Luteolin reduces IL-6 production in microglia by inhibiting JNK phosphorylation and activation of AP-1. Proc Natl Acad Sci U S A 2008;105:7534-9.

33. Chen X, Wu S, Chen C, et al. Omega-3 polyunsaturated fatty acid supplementation attenuates microglial-induced inflammation by inhibiting the HMGB1/TLR4/NF- $\mathrm{KB}$ pathway following experimental traumatic brain injury. $\mathrm{J}$ Neuroinflammation 2017;14:143.

34. Loddick SA, Turnbull AV, Rothwell NJ. Cerebral interleukin-6 is neuroprotective during permanent focal cerebral ischemia in the rat. J Cereb Blood Flow Metab 1998;18:176-9.

35. Bhat NR, Zhang P, Lee JC, et al. Extracellular signalregulated kinase and $\mathrm{p} 38$ subgroups of mitogen-activated protein kinases regulate inducible nitric oxide synthase and tumor necrosis factor-alpha gene expression in endotoxin-stimulated primary glial cultures. J Neurosci 1998;18:1633-41.

36. Waetzig V, Czeloth K, Hidding U, et al. c-Jun N-terminal kinases (JNKs) mediate pro-inflammatory actions of microglia. Glia 2005;50:235-46.

37. Ganbold T, Bao Q, Zandan J, et al. Modulation of Microglia Polarization through Silencing of NF-kB p65 by Functionalized Curdlan Nanoparticle-Mediated RNAi. ACS Appl Mater Interfaces 2020;12:11363-74.

38. Zheng Z, Kim JY, Ma H, et al. Anti-inflammatory effects of the $70 \mathrm{kDa}$ heat shock protein in experimental stroke. J Cereb Blood Flow Metab 2008;28:53-63.

39. Kim JB, Han AR, Park EY, et al. Inhibition of LPSinduced iNOS, COX-2 and cytokines expression by poncirin through the NF-kappaB inactivation in RAW 264.7 macrophage cells. Biol Pharm Bull 2007;30:2345-51.

40. Meng H, Zhao HR, Cao X, et al. Double-negative T cells remarkably promote neuroinflammation after ischemic stroke. Proc Natl Acad Sci U S A 2019;116:5558-63.

41. Pan J, Jin JL, Ge HM, et al. Malibatol A regulates microglia M1/M2 polarization in experimental stroke in a PPAR $\gamma$-dependent manner. J Neuroinflammation 2015;12:51. 